\title{
Uniformly Alpha-Quasi-Convex Functions Defined by Janowski Functions
}

\author{
Shahid Mahmood $\left(\mathbb{D},{ }^{1}\right.$ Sarfraz Nawaz Malik $\left(\mathbb{D},{ }^{2}\right.$ Sumbal Farman, ${ }^{2}$ \\ S. M. Jawwad Riaz, ${ }^{2}$ and Shabieh Farwa ${ }^{2}{ }^{2}$ \\ ${ }^{1}$ Department of Mechanical Engineering, Sarhad University of Science and Information Technology, Ring Road, Peshawar, Pakistan \\ ${ }^{2}$ Department of Mathematics, COMSATS Institute of Information Technology, Wah Cantt, Pakistan
}

Correspondence should be addressed to Sarfraz Nawaz Malik; snmalik110@yahoo.com

Received 25 October 2017; Revised 30 November 2017; Accepted 18 December 2017; Published 2 January 2018

Academic Editor: Hugo Leiva

Copyright (c) 2018 Shahid Mahmood et al. This is an open access article distributed under the Creative Commons Attribution License, which permits unrestricted use, distribution, and reproduction in any medium, provided the original work is properly cited.

In this work, we aim to introduce and study a new subclass of analytic functions related to the oval and petal type domain. This includes various interesting properties such as integral representation, sufficiency criteria, inclusion results, and the convolution properties for newly introduced class.

\section{Introduction and Preliminaries}

Let $\mathscr{A}$ be the class of functions $f(z)$ of the form

$$
f(z)=z+\sum_{n=2}^{\infty} a_{n} z^{n}
$$

which are analytic in the open unit disk $\mathscr{U}=\{z:|z|<1\}$. Let $\mathcal{S}, \mathcal{S}^{*}(\gamma), \mathscr{C}(\gamma)$, and $\mathscr{K}(\gamma)$ be the subclasses of $\mathscr{A}$ consisting of functions which are univalent, starlike of order $\gamma$, convex of order $\gamma$, and close-to-convex of order $\gamma$, respectively, $0 \leq$ $\gamma<1$. Clearly $\mathcal{S}^{*}(0)=\mathcal{S}^{*}$, the class of starlike univalent functions, $\mathscr{C}(0)=\mathscr{C}$, the class of convex univalent functions, and $\mathscr{K}(0)=\mathscr{K}$, the class of close-to-convex univalent functions.

For analytic functions $f(z)=z+\sum_{n=2}^{\infty} a_{n} z^{n}$ and $g(z)=z+$ $\sum_{n=2}^{\infty} b_{n} z^{n}$, the convolution or Hadamard product is denoted by $f * g$ and is defined by

$$
(f * g)(z)=z+\sum_{n=2}^{\infty} a_{n} b_{n} z^{n} .
$$

A function $f(z)$ is said to be subordinate to the function $g(z)$, written symbolically as $f(z) \prec g(z)$, if there exists a Schwarz function $w(z)$ such that

$$
f(z)=g(w(z)), \quad z \in \mathscr{U},
$$

where $w(0)=0,|w(z)|<1$ for $z \in \mathscr{U}$. The class $\mathscr{P}[A, B]$ consists of functions $f(z)$ which are analytic in $\mathcal{U}$ with $f(0)=$ 1 and

$$
f(z)<\frac{1+A z}{1+B z},
$$

where $-1 \leq B<A \leq 1$. If a function $f(z)$ belongs to $\mathscr{P}[A, B]$, then it maps $\mathcal{U}$ on to the domain $\Omega[A, B]$, defined by

$$
\Omega[A, B]=\left\{w:\left|w-\frac{1-A B}{1-B^{2}}\right|<\frac{A-B}{1-B^{2}}\right\} .
$$

The domain $\Omega[A, B]$ represents an open circular disk, having center at real axis and whose diameter points are $d_{1}=(1-$ $A) /(1-B)$ and $d_{2}=(1+A) /(1+B)$. For $B=-1$ and $A=1$, the class $\mathscr{P}[A, B]$ reduces to the well-known class $\mathscr{P}$ of Carathèodory functions; see [1]. The class $\mathscr{P}[A, B]$ is connected with the class $\mathscr{P}$ of Carathèodory functions by the following relation.

$$
f(z) \in \mathscr{P} \Longleftrightarrow \frac{(A+1) f(z)-(A-1)}{(B+1) f(z)-(B-1)} \in \mathscr{P}[A, B] .
$$

Janowski [2] introduced the classes $\mathcal{S}^{*}[A, B]$ and $\mathscr{C}[A, B]$ and studied them comprehensively. He established the Alexander type relation between these classes. 
In 1999, Kanas and Wiśniowska [3] gave the concept of general conic domain by introducing a domain $\Omega_{\beta}$, defined by

$$
\Omega_{\beta}=\left\{u+i v: u>\beta \sqrt{(u-1)^{2}+v^{2}}\right\} .
$$

The domain $\Omega_{\beta}$ represents a right half plane when $\beta=0$, the hyperbolic regions for $0<\beta<1$, a parabolic region for $\beta=1$, and it represents elliptic regions when $\beta>1$. The extremal function for conic domain $\Omega_{\beta}$ is the mapping $p_{\beta}(z)$ with $p_{\beta}(0)=1$ and $p_{\beta}^{\prime}(0)>0$ and is defined by

$$
p_{\beta}(z)= \begin{cases}\frac{1+z}{1-z}, & \beta=0, \\ 1+\frac{2}{\pi^{2}}\left(\log \frac{1+\sqrt{z}}{1-\sqrt{z}}\right)^{2}, & \beta=1, \\ 1+\frac{2}{1-\beta^{2}} \sin h^{2}\left[\left(\frac{2}{\pi} \arccos \beta\right) \arctan h \sqrt{z}\right], & 0<\beta<1, \\ 1+\frac{1}{\beta^{2}-1} \sin \left(\frac{\pi}{2 X(t)} \int_{0}^{u(z) / t} \frac{1}{\sqrt{1-x^{2}} \sqrt{1-(t x)^{2}}} d x\right)+\frac{1}{\beta^{2}-1}, & \beta>1,\end{cases}
$$

where $u(z)=(z-\sqrt{t}) /(1-\sqrt{t z}), t \in(0,1), z \in \mathcal{U}$, and $z$ is chosen such that $\beta=\cosh \left(\pi X^{\prime}(t) / 4 X(t)\right), X(t)$ is Legendre's complete elliptic integral of first kind, and $X^{\prime}(t)$ is complementary integral of $X(t)$; for more details, see [3]. Meanwhile, Kanas and Wiśniowska [3,4] introduced a class $\mathscr{P}\left(p_{\beta}\right)$ which maps $\mathcal{U}$ on to the conic regions, defined by the domain $\Omega_{\beta}$. They also defined the classes $\beta-\mathscr{U} \mathscr{C} \mathscr{V}$ and $\beta-\mathcal{S} \mathscr{T}$ of $\beta$-uniformly convex functions and their corresponding $\beta$-starlike functions; see $[3,4]$. Recently Noor and Malik [5] introduced new geometrical structures of oval and petal type shape as image domain and defined the class $\beta-\mathscr{P}[A, B]$ of functions which give these types of mappings. The concepts of Janowski functions and conic domains are combined together to define the class $\beta-\mathscr{P}[A, B]$ which represents the oval and petal type regions as image domain.

Definition 1 (see [5]). A function $p(z) \in \beta-\mathscr{P}[A, B]$ if and only if,

$$
p(z) \prec p_{\beta}(A, B ; z), \quad(\beta \geq 0,-1 \leq B<A \leq 1),
$$

where

$$
p_{\beta}(A, B ; z)=\frac{(A+1) p_{\beta}(z)-(A-1)}{(B+1) p_{\beta}(z)-(B-1)}
$$

with $p_{\beta}(z)$ as defined in (8).

Graphically, the function $p(z) \in \beta-\mathscr{P}[A, B]$ takes all the values in the domain $\Omega_{\beta}[A, B],-1 \leq B<A \leq 1$, which is defined as

$$
\begin{aligned}
& \Omega_{\beta}[A, B]=\left\{w(z): \operatorname{Re}\left(\frac{(B-1) w(z)-(A-1)}{(B+1) w(z)-(A+1)}\right)\right. \\
& \left.>\beta\left|\frac{(B-1) w(z)-(A-1)}{(B+1) w(z)-(A+1)}-1\right|\right\}
\end{aligned}
$$

or, equivalently,

$$
\begin{aligned}
\Omega_{\beta} & {[A, B]=\left\{u+i v:\left[\left(B^{2}-1\right)\left(u^{2}+v^{2}\right)\right.\right.} \\
& \left.-2(A B-1) u+\left(A^{2}+1\right)\right]^{2} \\
& >\left[-2(B+1)\left(u^{2}+v^{2}\right)+2(A+B+2) u\right. \\
& \left.\left.-2(A+1)^{2}\right]\right\} .
\end{aligned}
$$

Noor and Malik [5] also introduced two new classes $\beta-$ $\mathcal{U} \mathscr{C} \mathscr{V}[A, B]$ and $\beta-\mathcal{S} \mathscr{T}[A, B]$ related to the domain $\Omega_{\beta}[A, B]$ whose geometry is oval and petal type regions subject to the values of $A, B$, and beta. These classes are defined as follows.

Definition 2 (see [5]). A function $f(z) \in \mathscr{A}$ is said to be in the class $\beta-\mathscr{U} \mathscr{C} \mathscr{V}[A, B], k \geq 0,-1 \leq B<A \leq 1$, if and only if

$$
\begin{aligned}
& \operatorname{Re}\left(\frac{(B-1)\left(\left(z f^{\prime}(z)\right)^{\prime} / f^{\prime}(z)\right)-(A-1)}{(B+1)\left(\left(z f^{\prime}(z)\right)^{\prime} / f^{\prime}(z)\right)-(A+1)}\right) \\
& >\beta\left|\frac{(B-1)\left(\left(z f^{\prime}(z)\right)^{\prime} / f^{\prime}(z)\right)-(A-1)}{(B+1)\left(\left(z f^{\prime}(z)\right)^{\prime} / f^{\prime}(z)\right)-(A+1)}-1\right|,
\end{aligned}
$$

or, equivalently,

$$
\frac{\left(z f^{\prime}(z)\right)^{\prime}}{f^{\prime}(z)} \in \beta-\mathscr{P}[A, B] .
$$


Definition 3 (see [5]). A function $f(z) \in \mathscr{A}$ is said to be in the class $\beta-\mathcal{S} \mathscr{T}[A, B], k \geq 0,-1 \leq B<A \leq 1$, if and only if

$$
\begin{aligned}
& \operatorname{Re}\left(\frac{(B-1)\left(z f^{\prime}(z) / f(z)\right)-(A-1)}{(B+1)\left(z f^{\prime}(z) / f(z)\right)-(A+1)}\right) \\
& \quad>\beta\left|\frac{(B-1)\left(z f^{\prime}(z) / f(z)\right)-(A-1)}{(B+1)\left(z f^{\prime}(z) / f(z)\right)-(A+1)}-1\right|
\end{aligned}
$$

or, equivalently,

$$
\frac{z f^{\prime}(z)}{f(z)} \in \beta-\mathscr{P}[A, B] .
$$

Motivated from the recent work presented by Noor and Malik [5], we define the following new subclass of analytic functions associated with domain $\Omega_{\beta}[A, B]$.

Definition 4. A function $f(z) \in \mathscr{A}$ is said to be in the class $\beta-\mathscr{U} Q_{\alpha}[A, B, C, D], \beta \geq 0,0 \leq \alpha \leq 1,-1 \leq B<A \leq 1$, $-1 \leq D<C \leq 1$, if and only if

$$
\begin{aligned}
& \operatorname{Re}\left(\frac{(B-1) w(z)-(A-1)}{(B+1) w(z)-(A+1)}\right) \\
& \quad>\beta\left|\frac{(B-1) w(z)-(A-1)}{(B+1) w(z)-(A+1)}-1\right|,
\end{aligned}
$$

or

$$
w(z) \in \beta-\mathscr{P}[A, B]
$$

where

$$
w(z)=(1-\alpha) \frac{f^{\prime}(z)}{g^{\prime}(z)}+\alpha \frac{\left(z f^{\prime}(z)\right)^{\prime}}{g^{\prime}(z)}
$$

for some $g(z) \in \beta-\mathscr{U} \mathscr{C} \mathscr{V}[C, D]$.

It follows from the above definition that $f(z) \in \beta-$ $\mathcal{U} Q_{\alpha}[A, B, C, D]$ if and only if

$$
\begin{aligned}
F(z) & =(1-\alpha) f(z)+\alpha z f^{\prime}(z) \\
& \in \beta-\mathcal{U} \mathscr{K}[A, B, C, D],
\end{aligned}
$$

where $\beta-\mathscr{U} \mathscr{K}[A, B, C, D]$ is the class of uniformly Janowski close-to-convex functions, introduced and studied by Mahmood et al. [6].

Special Cases. By setting certain values to different parameters, the class $\beta-\mathcal{U} Q_{\alpha}[A, B, C, D]$ coincides with several renowned classes of analytic functions, as given below.

(1) $\beta-\mathscr{U} Q_{0}[A, B, C, D]=\beta-\mathscr{U} \mathscr{K}[A, B, C, D]$, $\beta-\mathscr{U} Q_{1}[A, B, C, D]=\beta-\mathscr{U Q}[A, B, C, D]$ are well-known classes of uniformly Janowski close-toconvex and uniformly Janowski quasi-convex functions respectively, introduced and studied by Mahmood et al. [6] .
(2) $\beta-\mathscr{U} Q_{\alpha}[1,-1,1,-1]=\beta-\mathscr{U} \mathscr{K}$ is the well known class of uniformly close-to-convex functions, introduced and studied by Noor et al. [7].

(3) $0-\mathscr{U} Q_{\alpha}[1,-1,1,-1]=\mathscr{Q}_{\alpha}$ is the well known class of alpha-quasi-convex functions, introduced and studied by Noor and Aboudi [8].

(4) $0-\mathscr{U} \mathbb{Q}_{\alpha}[A, B, 1,-1]=\mathbb{Q}_{\alpha}[A, B]$ is the well known class of alpha Janowski convex functions, introduced and studied by Selvaraj and Thirupathi [9].

Throughout this paper, we assume that $\beta \geq 0,-1 \leq B<A \leq$ $1,-1 \leq D<C \leq 1$, and $0 \leq \alpha \leq 1$ unless otherwise stated.

\section{A Set of Lemmas}

To prove our main results we need the following Lemmas.

Lemma 5 (see [5]). Let $p(z)=1+\sum_{n=1}^{\infty} c_{n} z^{n} \in \beta-\mathscr{P}[A, B]$. Then

$$
\left|c_{n}\right| \leq|\delta(A, B, \beta)|
$$

where

$$
\begin{aligned}
& \delta(A, B, \beta)=\frac{(A-B) \delta_{\beta}}{2}, \\
& \delta_{\beta}= \begin{cases}\frac{8\left(\cos ^{-1} \beta\right)^{2}}{\pi^{2}\left(1-\beta^{2}\right)}, & 0 \leq \beta<1, \\
\frac{8}{\pi^{2}}, & \beta=1, \\
\frac{\pi^{2}}{4 \sqrt{t}\left(\beta^{2}-1\right) R^{2}(t)(1+t)}, & \beta>1 .\end{cases}
\end{aligned}
$$

Lemma 6 (see [5]). Let $g(z) \in \beta-\mathscr{C} \mathscr{V}[C, D]$ with $\beta \geq 0$ and be given by

$$
g(z)=z+\sum_{n=2}^{\infty} b_{n} z^{n}
$$

Then

$$
\left|b_{n}\right| \leq \frac{1}{n} \prod_{j=0}^{n-2} \frac{\left|(C-D) \delta_{\beta}-2 j D\right|}{2(j+1)}
$$

where $\delta_{\beta}$ is defined by (23).

Lemma 7 (see [10]). Let $f(z)$ and $g(z)$ be in the classes $\mathscr{C}$ and $\mathcal{S}^{*}$, respectively. Then for every function $F(z)$ analytic in $\mathcal{U}$ with $F(0)=1$, we have

$$
\frac{f(z) * g(z) F(z)}{f(z) * g(z)} \in \overline{\mathrm{co}}(F(\mathcal{U})), \quad z \in \mathcal{U},
$$

where $\overline{\mathrm{co}} F(\mathcal{U})$ denotes the closed convex hull $F(\mathcal{U})$.

Lemma 8 (see [6]). Let $f(z) \in \beta-\mathscr{U} \mathscr{K}[A, B, C, D]$ and $\varphi(z) \in \mathscr{C}$. Then $(\varphi * f)(z) \in \beta-\mathscr{U} \mathscr{K}[A, B, C, D]$. 


\section{Main Results}

Theorem 9. Let $f(z) \in \beta-\mathscr{U} Q_{\alpha}[A, B, C, D]$. Then, the function $F(z)$ is defined such that

$$
f(z)=\frac{1}{\alpha} z^{1-1 / \alpha} \int_{0}^{z} t^{2-1 / \alpha} F(t) d t, \quad(\alpha>0, z \in \mathcal{U})
$$

belongs to the class $\beta-\mathscr{U} \mathscr{K}[A, B, C, D]$.

Proof. From (27), we can write

$$
(1-\alpha) f(z)+\alpha z f^{\prime}(z)=F(z), \quad(z \in \mathcal{U})
$$

and the result follows by using (20).

Theorem 10. A function $f(z)$ having the form (1) is in the class $\beta-\mathcal{U} Q_{\alpha}[A, B, C, D]$ if it satisfies

$$
\left[2 ( \beta + 1 ) \left(\sum_{n=2}^{\infty} \prod_{j=0}^{n-2} \frac{\left|\delta_{\beta}(C-D)-2 j D\right|}{2(j+1)}\right.\right.
$$

$$
\begin{aligned}
& \left.+\sum_{n=2}^{\infty} n(1+\alpha(n-1))\left|a_{n}\right|\right) \\
& +\sum_{n=2}^{\infty}|B+1| n(1+\alpha(n-1))\left|a_{n}\right| \\
& \left.+\sum_{n=2}^{\infty} \prod_{j=0}^{n-2} \frac{\left|\delta_{\beta}(C-D)-2 j D\right|}{2(j+1)}|A+1|\right]<|B-A| .
\end{aligned}
$$

Proof. Assuming that (29) holds true, then it is sufficient to show that

$$
\begin{aligned}
& \beta\left|\frac{(B-1) w(z)-(A-1)}{(B+1) w(z)-(A+1)}-1\right| \\
& -\operatorname{Re}\left[\frac{(B-1) w(z)-(A-1)}{(B+1) w(z)-(A+1)}-1\right]<1 .
\end{aligned}
$$

Now consider

$$
\begin{aligned}
& \beta\left|\frac{(B-1)\left((1-\alpha)\left(f^{\prime}(z) / g^{\prime}(z)\right)+\alpha\left(\left(z f^{\prime}(z)\right)^{\prime} / g^{\prime}(z)\right)\right)-(A-1)}{(B+1)\left((1-\alpha)\left(f^{\prime}(z) / g^{\prime}(z)\right)+\alpha\left(\left(z f^{\prime}(z)\right)^{\prime} / g^{\prime}(z)\right)\right)-(A+1)}-1\right| \\
& -\operatorname{Re}\left[\frac{(B-1)\left((1-\alpha)\left(f^{\prime}(z) / g^{\prime}\right)+\alpha\left(\left(z f^{\prime}(z)\right)^{\prime} / g^{\prime}\right)\right)-(A-1)}{(B+1)\left((1-\alpha)\left(f^{\prime}(z) / g^{\prime}(z)\right)+\alpha\left(\left(z f^{\prime}(z)\right)^{\prime} / g^{\prime}(z)\right)\right)-(A+1)}-1\right] \\
& \leq(\beta+1)\left|\frac{(B-1)\left[f^{\prime}(z) / g^{\prime}(z)+\alpha\left(z f^{\prime \prime}(z) / g^{\prime}(z)\right)\right]-(A-1)}{(B+1)\left[f^{\prime}(z) / g^{\prime}(z)+\alpha\left(z f^{\prime \prime}(z) / g^{\prime}(z)\right)\right]-(A+1)}-1\right| \\
& =(\beta+1)\left|\frac{(B-1)\left[f^{\prime}(z)+\alpha z f^{\prime \prime}(z)\right]-(A-1) g^{\prime}(z)}{(B+1)\left[f^{\prime}(z)+\alpha z f^{\prime \prime}(z)\right]-(A+1) g^{\prime}(z)}-1\right| \\
& =(\beta+1)\left|\frac{(B-1)\left[f^{\prime}(z)+\alpha z f^{\prime \prime}(z)\right]-(A-1) g^{\prime}(z)-(B+1)\left[f^{\prime}(z)+\alpha z f^{\prime \prime}(z)\right]+(A+1) g^{\prime}(z)}{(B+1)\left[f^{\prime}(z)+\alpha z f^{\prime \prime}(z)\right]-(A+1) g^{\prime}(z)}\right| \\
& =2(\beta+1)\left|\frac{g^{\prime}(z)-f^{\prime}(z)-\alpha z f^{\prime \prime}(z)}{(B+1)\left[f^{\prime}(z)+\alpha z f^{\prime \prime}(z)\right]-(A+1) g^{\prime}(z)}\right| \\
& \leq 2(\beta+1)\left|\frac{1+\sum_{n=2}^{\infty} n b_{n} z^{n-1}-1-\sum_{n=2}^{\infty} n a_{n} z^{n-1}-\alpha \sum_{n=2}^{\infty} n(n-1) a_{n} z^{n-1}}{(B+1)\left(1+\sum_{n=2}^{\infty} n a_{n} z^{n-1}-\alpha \sum_{n=2}^{\infty} n(n-1) a_{n} z^{n-1}\right)-(A+1)\left(1+\sum_{n=2}^{\infty} n b_{n} z^{n-1}\right)}\right| \\
& =2(\beta+1)\left|\frac{\sum_{n=2}^{\infty} n b_{n} z^{n-1}-\sum_{n=2}^{\infty} n(1+\alpha(n-1)) a_{n} z^{n-1}}{(B-A)+(B+1)\left(\sum_{n=2}^{\infty} n(1+\alpha n-\alpha) a_{n} z^{n-1}\right)-(A+1)\left(\sum_{n=2}^{\infty} n b_{n} z^{n-1}\right)}\right| \\
& \leq 2(\beta+1) \frac{\sum_{n=2}^{\infty} n\left|b_{n}\right|+\sum_{n=2}^{\infty} n(1+\alpha(n-1))\left|a_{n}\right|}{|B-A|-\left|(B+1)\left(\sum_{n=2}^{\infty} n(1+\alpha n-\alpha) a_{n} z^{n-1}\right)-(A+1)\left(\sum_{n=2}^{\infty} n b_{n} z^{n-1}\right)\right|} \\
& \leq 2(\beta+1) \frac{\sum_{n=2}^{\infty} n\left|b_{n}\right|+\sum_{n=2}^{\infty} n(1+\alpha(n-1))\left|a_{n}\right|}{|B-A|-|B+1| \sum_{n=2}^{\infty} n(1+\alpha(n-1))\left|a_{n}\right|-|A+1| \sum_{n=2}^{\infty} n\left|b_{n}\right|} \\
& \leq 2(\beta+1) \frac{\sum_{n=2}^{\infty} \prod_{j=0}^{n-2}\left(\left|\delta_{\beta}(C-D)-2 j D\right| / 2(j+1)\right)+\sum_{n=2}^{\infty} n(1+\alpha(n-1))\left|a_{n}\right|}{|B-A|-|B+1| \sum_{n=2}^{\infty} n(1+\alpha n-\alpha)\left|a_{n}\right|-|A+1| \sum_{n=2}^{\infty} \prod_{j=0}^{n-2}\left(\left|\delta_{\beta}(C-D)-2 j D\right| / 2(j+1)\right)} .
\end{aligned}
$$


In the last inequality, we have used the following coefficient inequality for functions belonging to the class $\beta-\mathscr{C} \mathscr{V}[C, D]$ (see [5]):

$$
\left|b_{n}\right| \leq \frac{1}{n} \prod_{j=0}^{n-2} \frac{\left|\delta_{\beta}(C-D)-2 j D\right|}{2(j+1)}
$$

The last inequality is bounded above by 1 , if

$$
\begin{aligned}
& {\left[2 ( \beta + 1 ) \left(\sum_{n=2}^{\infty} \prod_{j=0}^{n-2} \frac{\left|\delta_{\beta}(C-D)-2 j D\right|}{2(j+1)}\right.\right.} \\
& \left.+\sum_{n=2}^{\infty} n(1+\alpha(n-1))\left|a_{n}\right|\right) \\
& +\sum_{n=2}^{\infty}|B+1| n(1+\alpha(n-1))\left|a_{n}\right| \\
& \left.+\sum_{n=2}^{\infty} \prod_{j=0}^{n-2} \frac{\left|\delta_{\beta}(C-D)-2 j D\right|}{2(j+1)}|A+1|\right]<|B-A| .
\end{aligned}
$$

When $\alpha=0$, Theorem 10 reduces to the following known result, proved in [6].

Corollary 11. If a function $f(z) \in \mathscr{A}$ satisfies

$$
\begin{aligned}
& {\left[2(\beta+1)\left(\sum_{n=2}^{\infty} \prod_{j=0}^{n-2} \frac{\left|\delta_{\beta}(C-D)-2 j D\right|}{2(j+1)}+\sum_{n=2}^{\infty} n\left|a_{n}\right|\right)\right.} \\
& +\sum_{n=2}^{\infty}|B+1| n\left|a_{n}\right| \\
& \left.+\sum_{n=2}^{\infty} \prod_{j=0}^{n-2} \frac{\left|\delta_{\beta}(C-D)-2 j D\right|}{2(j+1)}|A+1|\right]<|B-A|,
\end{aligned}
$$

then $f(z) \in \beta-\mathscr{U} \mathscr{K}[A, B, C, D]$.

When $\alpha=1$, Theorem 10 takes the form of following known result, proved in [6].

Corollary 12. A function $f(z) \in \mathscr{A}$ is said to be in the class $\beta-\mathcal{U Q}[A, B, C, D]$ if it satisfies the condition

$$
\begin{aligned}
& {\left[2(\beta+1)\left(\sum_{n=2}^{\infty} \prod_{j=0}^{n-2} \frac{\left|\delta_{\beta}(C-D)-2 j D\right|}{2(j+1)}+\sum_{n=2}^{\infty} n^{2}\left|a_{n}\right|\right)\right.} \\
& +\sum_{n=2}^{\infty}|B+1| n^{2}\left|a_{n}\right| \\
& \left.+\sum_{n=2}^{\infty} \prod_{j=0}^{n-2} \frac{\left|\delta_{\beta}(C-D)-2 j D\right|}{2(j+1)}|A+1|\right]<|B-A| .
\end{aligned}
$$

Theorem 13. Let $f(z) \in \beta-\mathcal{U} Q_{\alpha}[A, B, C, D]$ and be of the form (1). Then for $n \geq 2$, we have

$$
\begin{aligned}
& \left|a_{n}\right| \leq \frac{1}{[n+\alpha n(n-1)]}\left[\prod_{j=0}^{n-2} \frac{\left|\delta_{\beta}(C-D)-2 j D\right|}{2(j+1)}\right. \\
& \left.\quad+\delta(A, B, \beta) \sum_{j=1}^{n-1} \prod_{m=0}^{j-2} \frac{\left|\delta_{\beta}(C-D)-2 m D\right|}{2(m+1)}\right],
\end{aligned}
$$

where $\delta(A, B, \beta)$ is given by (22).

Proof. By definition, for $f(z) \in \beta-\mathscr{U}_{Q_{\alpha}}[A, B, C, D]$, we get

$$
(1-\alpha) \frac{f^{\prime}(z)}{g^{\prime}(z)}+\alpha \frac{\left(z f^{\prime}(z)\right)^{\prime}}{g^{\prime}(z)}=p(z)
$$

where

$$
p(z)=1+\sum_{n=1}^{\infty} c_{n} z^{n} \prec p_{\beta}(A, B ; z) .
$$

We can write (37) as

$$
f^{\prime}(z)-\alpha z f^{\prime \prime}(z)=p(z) g^{\prime}(z) .
$$

Now using the series expansion, (39) reduces to

$$
\begin{aligned}
1 & +\sum_{n=2}^{\infty} n a_{n} z^{n-1}+\alpha \sum_{n=2}^{\infty} n(n-1) a_{n} z^{n-1} \\
& =\left(1+\sum_{n=2}^{\infty} n b_{n} z^{n-1}\right)\left(1+\sum_{n=2}^{\infty} c_{n-1} z^{n-1}\right) .
\end{aligned}
$$

Equating coefficients of $z^{n-1}$ on both sides, one may get

$$
n a_{n}+\alpha n(n-1) a_{n}=n b_{n}+\sum_{j=1}^{n-1} j b_{j} c_{n-j} .
$$

This implies that

$$
\left|a_{n}\right|[n+\alpha n(n-1)] \leq n\left|b_{n}\right|+\sum_{j=1}^{n-1} j\left|b_{j}\right|\left|c_{n-j}\right| .
$$

Now applying Lemmas 5 and 6, (42) reduces to

$$
\begin{aligned}
& \left|a_{n}\right| \leq \frac{1}{[n+\alpha n(n-1)]}\left[\prod_{j=0}^{n-2} \frac{\left|\delta_{\beta}(C-D)-2 j D\right|}{2(j+1)}\right. \\
& \left.\quad+\delta(A, B, \beta) \sum_{j=1}^{n-1} \prod_{m=0}^{j-2} \frac{\left|\delta_{\beta}(C-D)-2 m D\right|}{2(m+1)}\right],
\end{aligned}
$$

where $\delta(A, B, \beta)$ is given by (22). This completes the proof.

By setting $\alpha=0$ in Theorem 13, we have the following known result, proved by Mahmood et al. [6]. 
Corollary 14. Let $f(z) \in \beta-\mathscr{U} \mathscr{K}[A, B, C, D]$ and $a_{n}$ be the nth coefficient of Taylor series of $f(z)$. Then, for $n \geq 2$, we obtain

$$
\begin{gathered}
\left|a_{n}\right| \leq \frac{1}{n}\left[\prod_{j=0}^{n-2} \frac{\left|\delta_{\beta}(C-D)-2 j D\right|}{2(j+1)}+\delta(A, B, \beta)\right. \\
\left.\cdot \sum_{j=1}^{n-1} \prod_{m=0}^{j-2} \frac{\left|\delta_{\beta}(C-D)-2 m D\right||A-B| \delta_{\beta}}{2(m+1)}\right],
\end{gathered}
$$

where $\delta(A, B, \beta)$ is given by (22).

For $\alpha=1$, Theorem 13 takes the form of following known result, proved by Mahmood et al. [6].

Corollary 15. Let the function $f(z)$ of the form (1) belong to the class $\beta-\mathcal{U} Q[A, B, C, D]$. Then,

$$
\begin{gathered}
\left|a_{n}\right| \leq \frac{1}{n^{2}}\left[\prod_{j=0}^{n-2} \frac{\left|\delta_{\beta}(C-D)-2 j D\right|}{2(j+1)}+\delta(A, B, \beta)\right. \\
\left.\cdot \sum_{j=1}^{n-1} \prod_{m=0}^{j-2} \frac{\left|\delta_{\beta}(C-D)-2 m D\right||A-B| \delta_{\beta}}{2(m+1)}\right],
\end{gathered}
$$

where $n \geq 2$ and $\delta(A, B, \beta)$ is given by (22).

When we consider $A=C=1, B=D=-1$, and $\alpha=0$ in Theorem 13, we obtain the following known result, proved by Noor et al. [7].

Corollary 16. Let $f(z) \in \beta-\mathscr{U K}$ and $a_{n}$ be the $n$th coefficient of Taylor series of $f(z)$. Then,

$$
\left|a_{n}\right| \leq \frac{\left(\left|\delta_{\beta}\right|\right)_{n-1}}{n !}+\frac{\delta_{\beta}}{n} \sum_{j=0}^{n-1} \frac{\left(\left|\delta_{\beta}\right|\right)_{j-1}}{(j-1) !}, \quad(n \geq 2) .
$$

By Putting $\beta=0, C=1$, and $D=-1$ in Theorem 13, we have the following known result proved by Selvaraj and Thirupathi [9].

Corollary 17. Let $f(z) \in \mathbb{Q}_{\alpha}[A, B]$ and be of the form (1). Then,

$$
\begin{aligned}
& \left|a_{n}\right| \leq \frac{1}{1+\alpha(n-1)}\left[1+\frac{(A-B)(n-1)}{2}\right], \\
& (n \geq 2) \text {. }
\end{aligned}
$$

When we take $A=C=1, B=D=-1$, and $\beta=0$ in Theorem 13, we obtain the following known result proved by Noor and Aboudi [8].

Corollary 18. Let $f(z) \in \mathbb{Q}_{\alpha}[A, B]$ and $a_{n}$ be the nth coefficient of Taylor series of $f(z)$. Then,

$$
\left|a_{n}\right| \leq \frac{n}{1+\alpha(n-1)}, \quad(n \geq 2) .
$$

Theorem 19. Let $f(z) \in \beta-\mathscr{U Q}_{\alpha}[A, B, C, D], \alpha \geq 0$, and $\phi(z)$ be any convex univalent function in $\mathcal{U}$. Then

$$
f(z) * \phi(z) \in \beta-\mathscr{U} \mathbb{Q}_{\alpha}[A, B, C, D], \quad(z \in \mathcal{U}) .
$$

Proof. Let $f(z) \in \beta-\mathscr{U} Q_{\alpha}[A, B, C, D], \alpha \geq 0$. Then by (20), we have

$$
(1-\alpha) f(z)+\alpha z f^{\prime}(z) \in \beta-\mathscr{U} \mathscr{K}[A, B, C, D] .
$$

Using Lemma 8, one may have

$$
\begin{aligned}
& \left((1-\alpha) f(z)+\alpha z f^{\prime}(z)\right) * \phi(z) \\
& \quad \in \beta-\mathscr{U} \mathscr{K}[A, B, C, D],
\end{aligned}
$$

which implies that

$$
\begin{aligned}
& \left((1-\alpha)(f * \phi)(z)+\alpha z(f * \phi)^{\prime}(z)\right) \\
& \quad \in \beta-\mathscr{U} \mathscr{K}[A, B, C, D] .
\end{aligned}
$$

Hence by $(20), f(z) * \phi(z) \in \beta-\mathcal{U} Q_{\alpha}[A, B, C, D]$. This completes the proof.

\section{Applications of Theorem 19}

Theorem 20. Let $f(z) \in \beta-\mathscr{U} Q_{\alpha}[A, B, C, D]$. Then the functions $\phi_{i}(z)$, such that

$$
\begin{aligned}
& \phi_{1}(z)=\int_{0}^{z} \frac{f(t)}{t} d t, \\
& \phi_{2}(z)=\frac{2}{z} \int_{0}^{z} f(t) d t, \\
& \phi_{3}(z)=\int_{0}^{z} \frac{f(t)-f(t)}{t-t x} d t, \quad|x| \leq 1, x \neq 1, \\
& \phi_{4}(z)=\frac{\lambda+1}{z^{\lambda}} \int_{0}^{z} t^{\lambda-1} f(t) d t, \quad \operatorname{Re}(\lambda)>0(z \in \mathcal{U}),
\end{aligned}
$$

belong to the class $\beta-\mathcal{U} \mathbb{Q}_{\alpha}[A, B, C, D]$.

Proof. The proof follows immediately, when we observe that

$$
\phi_{i}(z)=\left(f * \psi_{i}\right)(z), \quad i=1,2,3,4(z \in \mathcal{U}),
$$

where $\psi_{i}(z)$ is convex in $\mathcal{U}$ such that

$$
\begin{aligned}
& \psi_{1}=-\log (1-z)=\sum_{n=1}^{\infty} \frac{1}{n} z^{n}, \\
& \psi_{2}=\frac{-[z+\log (1-z)]}{z}, \\
& \psi_{3}=\frac{1}{1-x} \log \left(\frac{1-x z}{1-z}\right), \\
& \psi_{4}=\sum_{n=1}^{\infty} \frac{1+\lambda}{n+\lambda} z^{n}, \quad \operatorname{Re}(\lambda)>0,(z \in \mathcal{U}) .
\end{aligned}
$$


Theorem 21. For $\alpha \geq 0$, we have $\beta-\mathcal{U} Q_{\alpha}[A, B, C, D] \subseteq \beta-$ $\mathcal{U} \mathscr{K}[A, B, C, D]$.

Proof. For $\alpha=0$, the result is obvious. So we assume that $\alpha>0$ and using the integral representation for $f(z) \in \beta-$ $\mathcal{U} Q_{\alpha}[A, B, C, D]$, we have

$$
f(z)=\frac{1}{\alpha} z^{1-1 / \alpha} \int_{0}^{z} t^{2-1 / \alpha} F(t) d t, \quad(z \in \mathcal{U}),
$$

where $F(z) \in \beta-\mathscr{U} \mathscr{K}[A, B, C, D]$, since $f(z)=\phi_{\alpha}(z) * F(z)$, where

$$
\phi_{\alpha}(z)=\sum_{n=1}^{\infty} \frac{1}{\alpha(n-1)+1} z^{n}, \quad(z \in \mathscr{U})
$$

is a convex function in $\mathcal{U}$. Now using Lemma 8 , we conclude that $f(z) \in \beta-\mathscr{U} \mathscr{K}[A, B, C, D]$. This completes the proof.

Theorem 22. If $0 \leq \alpha_{1} \leq \alpha_{2}$, Then,

$$
\beta-\mathscr{U} Q_{\alpha_{2}}[A, B, C, D] \subset \beta-\mathscr{U} Q_{\alpha_{1}}[A, B, C, D] .
$$

Proof. Let $f(z) \in \beta-\mathscr{U} Q_{\alpha_{2}}[A, B, C . D]$. Then

$$
\left[\left(1-\alpha_{2}\right) \frac{f^{\prime}(z)}{g^{\prime}(z)}+\alpha_{2} \frac{\left(z f^{\prime}(z)\right)^{\prime}}{g^{\prime}(z)}\right] \in \beta-\mathscr{P}[A, B] .
$$

Now consider

$$
\begin{gathered}
j\left(\alpha_{1}, f ; z\right)=\left[\left(1-\alpha_{1}\right) \frac{f^{\prime}(z)}{g^{\prime}(z)}+\alpha_{1} \frac{\left(z f^{\prime}(z)\right)^{\prime}}{g^{\prime}(z)}\right] \\
=\left(1-\frac{\alpha_{1}}{\alpha_{2}}\right) \frac{f^{\prime}(z)}{g^{\prime}(z)} \\
+\frac{\alpha_{1}}{\alpha_{2}}\left[\left(1-\alpha_{2}\right) \frac{f^{\prime}(z)}{g^{\prime}(z)}+\alpha_{2} \frac{\left(z f^{\prime}(z)\right)^{\prime}}{g^{\prime}(z)}\right] \\
=\left(1-\frac{\alpha_{1}}{\alpha_{2}}\right) j(0, f ; z)+\frac{\alpha_{1}}{\alpha_{2}} j\left(\alpha_{2}, f ; z\right) .
\end{gathered}
$$

Since $\beta-\mathscr{P}[A, B]$ is convex,

$$
\left(1-\frac{\alpha_{1}}{\alpha_{2}}\right) j(0, f ; z)+\frac{\alpha_{1}}{\alpha_{2}} j\left(\alpha_{2}, f ; z\right) \in \beta-\mathscr{P}[A, B]
$$

and consequently $f(z) \in \beta-\mathscr{U} Q_{\alpha_{1}}[A, B, C, D]$. This completes the proof.

\section{Conflicts of Interest}

The authors declare that there are no conflicts of interest regarding the publication of this paper.

\section{Acknowledgments}

The authors would like to acknowledge Professor Dr. Salim ur Rehman, V.C. Sarhad University of Science \& IT, for providing excellent research and academic environment.

\section{References}

[1] A. W. Goodman, Univalent Functions, vol. 1-2, Polygonal Publishing House, Washington, DC, USA, 1983.

[2] W. Janowski, "Some extremal problems for certain families of analytic functions," Annales Polonici Mathematici, vol. 28, pp. 297-326, 1973.

[3] S. Kanas and W. Wiśniowska, "Conic regions and k-uniform convexity," Journal of Computational and Applied Mathematics, vol. 105, pp. 327-336, 1999.

[4] S. Kanas and W. Wiśniowska, "Conic domains and starlike functions," Revue Roumaine de Mathématiques Pures et Appliquées, vol. 45, pp. 647-657, 2000.

[5] K. I. Noor and S. N. Malik, "On coefficient inequalities of functions associated with conic domains," Computers \& Mathematics with Applications, vol. 62, pp. 2209-2217, 2011.

[6] S. Mahmood, M. Arif, and S. N. Malik, "Janowski type closeto-convex functions associated with conic regions," Journal of Inequalities and Applications, vol. 2017, article 259, 2017.

[7] K. I. Noor, M. Arif, and W. Ul-Haq, "On k-uniformly close-toconvex functions of complex order," Applied Mathematics and Computation, vol. 215, no. 2, pp. 629-635, 2009.

[8] K. I. Noor and A. Aboudi, "Alpha quasi convex univalent functions," Caribbean Journal of Mathematics, vol. 3, pp. 1-8, 1984.

[9] C. Selvaraj and G. Thirupathi, "Certain generalization of Noor functions," International Journal of Mathematical Analysis, vol. 9, no. 17, pp. 847-855, 2015.

[10] S. S. Miller and P. T. Mocanu, Differential Subordinations: Theory and Applications, Pure and Applied Mathematics no. 225, Dekker, New York, NY, USA, 2000. 


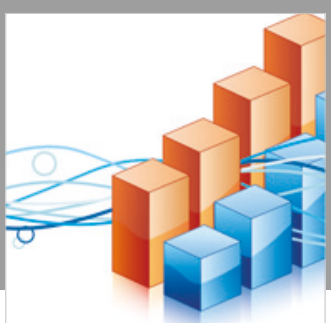

Advances in

Operations Research

\section{-n-m}
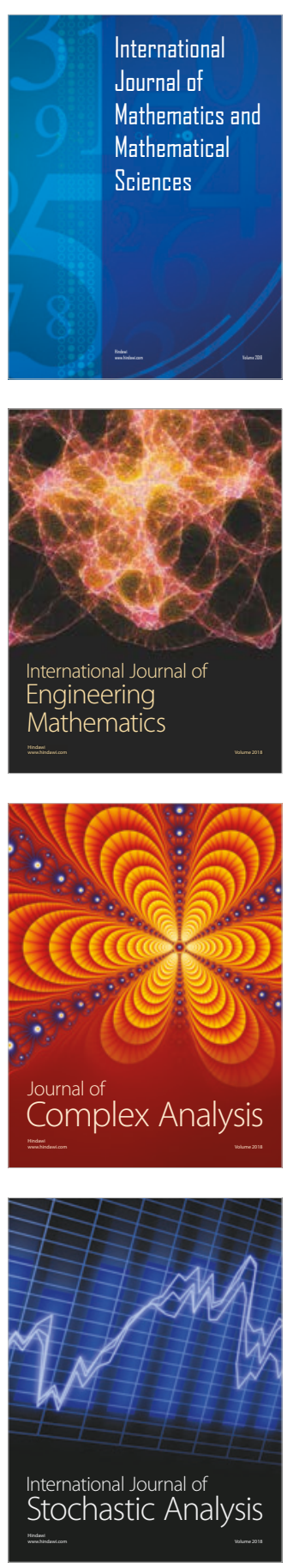
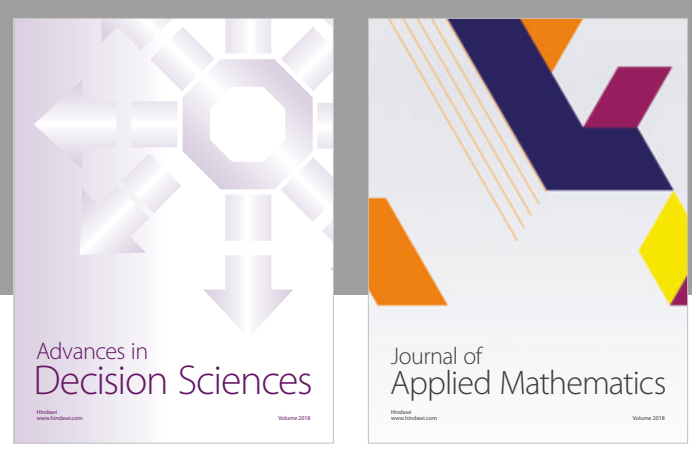

Journal of

Applied Mathematics
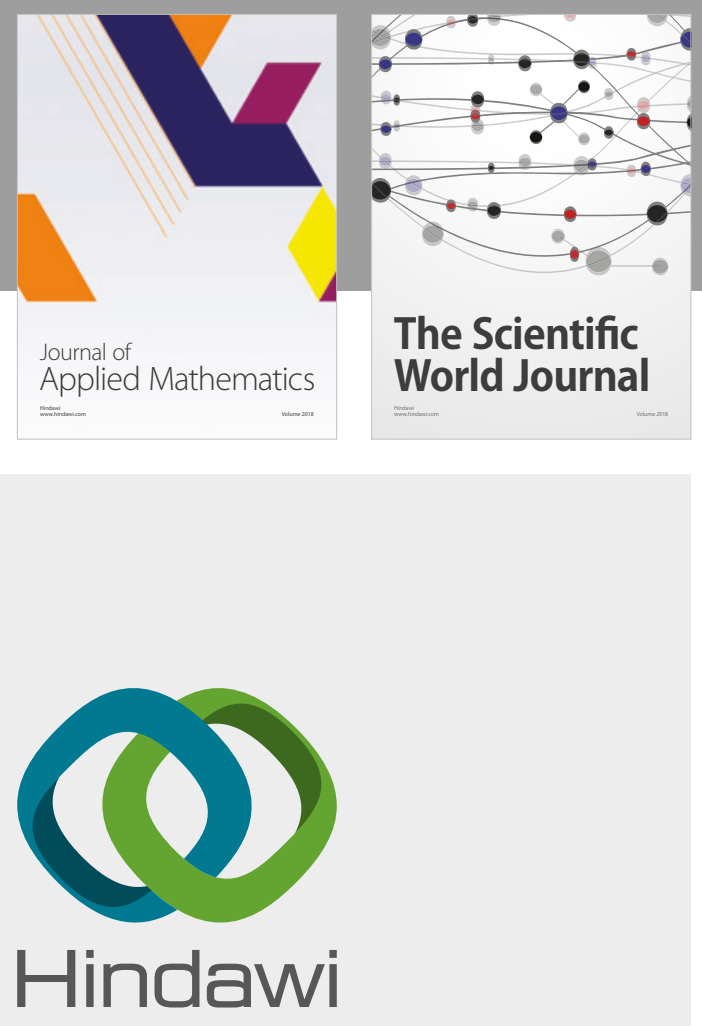

Submit your manuscripts at

www.hindawi.com

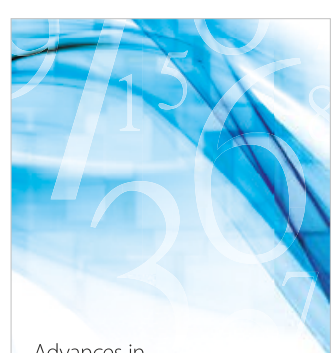

Advances in
Numerical Analysis
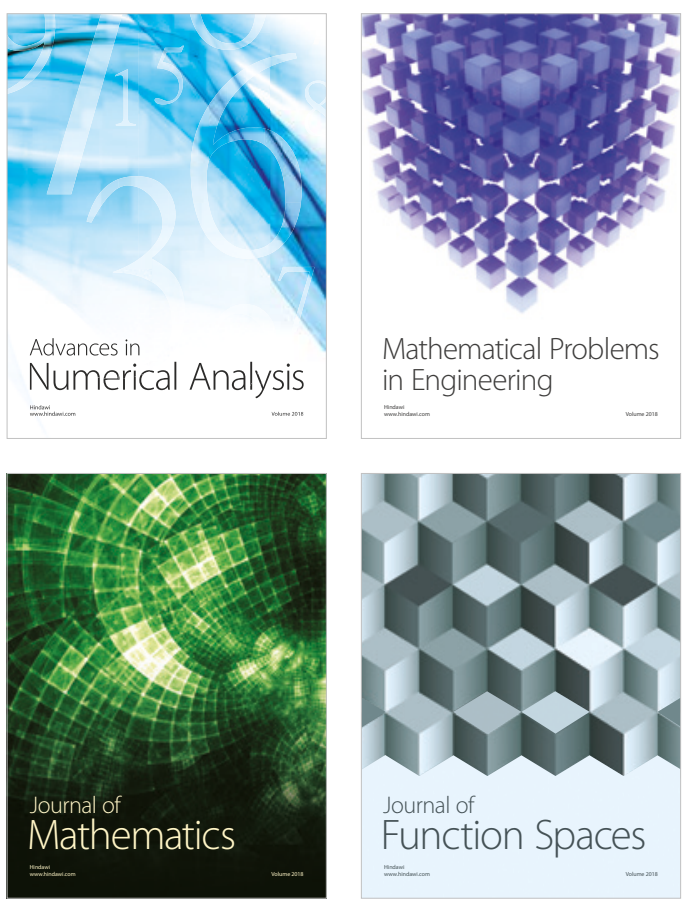

Mathematical Problems in Engineering

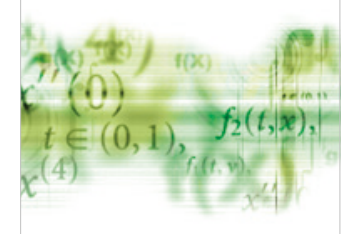

International Journal of

Differential Equations

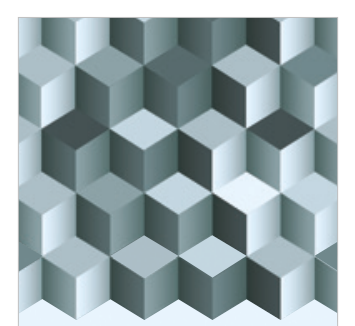

Journal of

Function Spaces

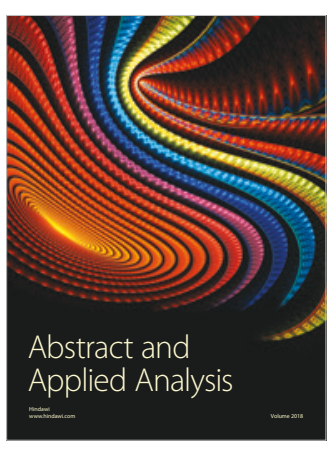

The Scientific

World Journal

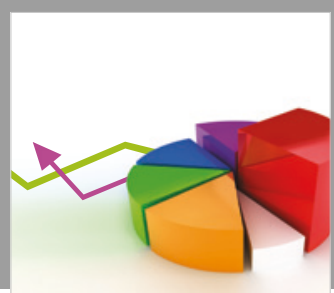

Journal of

Probability and Statistics
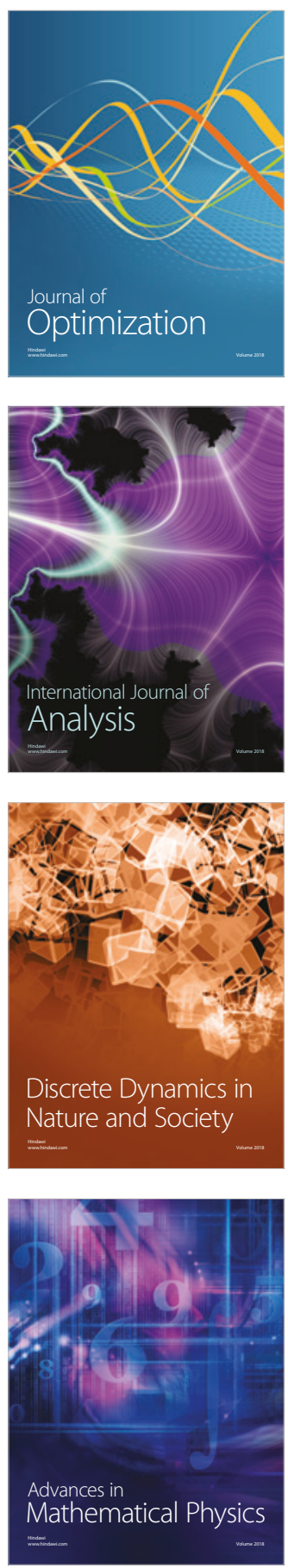